\title{
External Validation of the LabBM Score in Patients With Brain Metastases
}

\author{
Carsten Nieder ${ }^{\mathrm{a}, \mathrm{b}, \mathrm{c}}$, Astrid Dalhauga ${ }^{\mathrm{a}}$, Adam Pawinski ${ }^{\mathrm{a}}$
}

\begin{abstract}
Background: The aim of this study was to validate the prognostic impact of the recently introduced three-tiered LabBM score in patients with brain metastases. In contrast to the previous development and validation cohorts, the present cohort did not include patients treated with primary surgery and/or radiosurgery. The score is based on hemoglobin, platelet counts, albumin, C-reactive protein and lactate dehydrogenase.
\end{abstract}

Methods: This was a retrospective single institution analysis. Overall, 167 patients managed with first-line whole-brain radiotherapy (WBRT) were identified from a prospectively maintained database.

Results: The LabBM score significantly predicted overall survival (median 4.0, 2.9 and 1.5 months, respectively).

Conclusions: The LabBM score is also valid in a patient population that differs from the previously studied cohorts, that is patients who were judged to be better candidates for WBRT than surgery or radiosurgery. As these patients in general represent a less favorable subset, their median survival was shorter than reported in the development cohort (11, 7 and 3 months, respectively). Future studies should examine whether or not combinations of the LabBM and other scores, for example, lung-molGPA and melanoma-molGPA, improve the clinical value of single scores.

Keywords: Palliative radiotherapy; Whole brain radiotherapy; Brain metastases; Prognostic factors; Lactate dehydrogenase; Hemoglobin

\section{Introduction}

Treatment of patients with brain metastases is mainly per-

Manuscript submitted February 17, 2019, accepted March 16, 2019

\footnotetext{
aDepartment of Oncology and Palliative Medicine, Nordland Hospital, Bodo, Norway

bepartment of Clinical Medicine, Faculty of Health Sciences, UiT - The Arctic University of Norway, Tromso, Norway

${ }^{\mathrm{c} C}$ Corresponding Author: Carsten Nieder, Department of Oncology and Palliative Medicine, Nordland Hospital, 8092 Bodo, Norway.

Email: carsten.nieder@nlsh.no
}

doi: https://doi.org/10.14740/jocmr3746 formed with palliative aim and only few patients achieve long-term survival [1]. Ideally, patients with limited prognosis would be spared unnecessary burden of intense treatment, while those with better prognosis would receive the therapeutic measures required to prevent neurologic death in an attempt to translate optimal local control into long-term survival [2]. Several prognostic scores and nomograms have been developed to support pretreatment assessment [2-5]. Initially they were heavily based on performance status and extracranial disease extent or control [6]. Recently, additional tumor characteristics have been integrated, for example, in the molecular lung cancer score [7]. It has also been realized that blood biomarkers such as lactate dehydrogenase (LDH) and albumin [8], as well as hemoglobin may contribute to more accurate models predicting overall survival. In this context, Berghoff et al developed and validated a three-tiered score (LabBM), which includes a relatively high number of blood test results (hemoglobin, platelet count, albumin, LDH and C-reactive protein (CRP)) [9]. Median survival in the validation cohort was 10,6 and 1 month, respectively. Most patients in their study were managed with first-line stereotactic radiotherapy (SRS) or surgical resection. It is therefore necessary to analyze the score's performance in patients who received first-line whole-brain radiotherapy (WBRT), because they did not fulfill the selection criteria for SRS or resection, for example, number, size or location of the brain metastases. Most likely, the decision to recommend primary WBRT already points towards a relatively poor prognosis, resulting in uncertainty about the survival outcomes observed in the three LabBM classes after more aggressive local therapy. Therefore, we tested the LabBM score in a previously utilized, continuously updated database that includes patients treated with upfront WBRT [8, 10, 11].

\section{Materials and Methods}

A retrospective study of all patients with parenchymal brain metastases from histologically verified extracranial primary tumors treated with first-line WBRT at our hospital was performed. WBRT consisted of 10 fractions of 3 Gy. Further treatment was individualized and consisted of salvage SRS, systemic therapy or best supportive care (BSC). Systemic treatment was usually prescribed as judged appropriate by the patients' medical oncologists. The patients were treated be- 
Table 1. Patient Characteristics

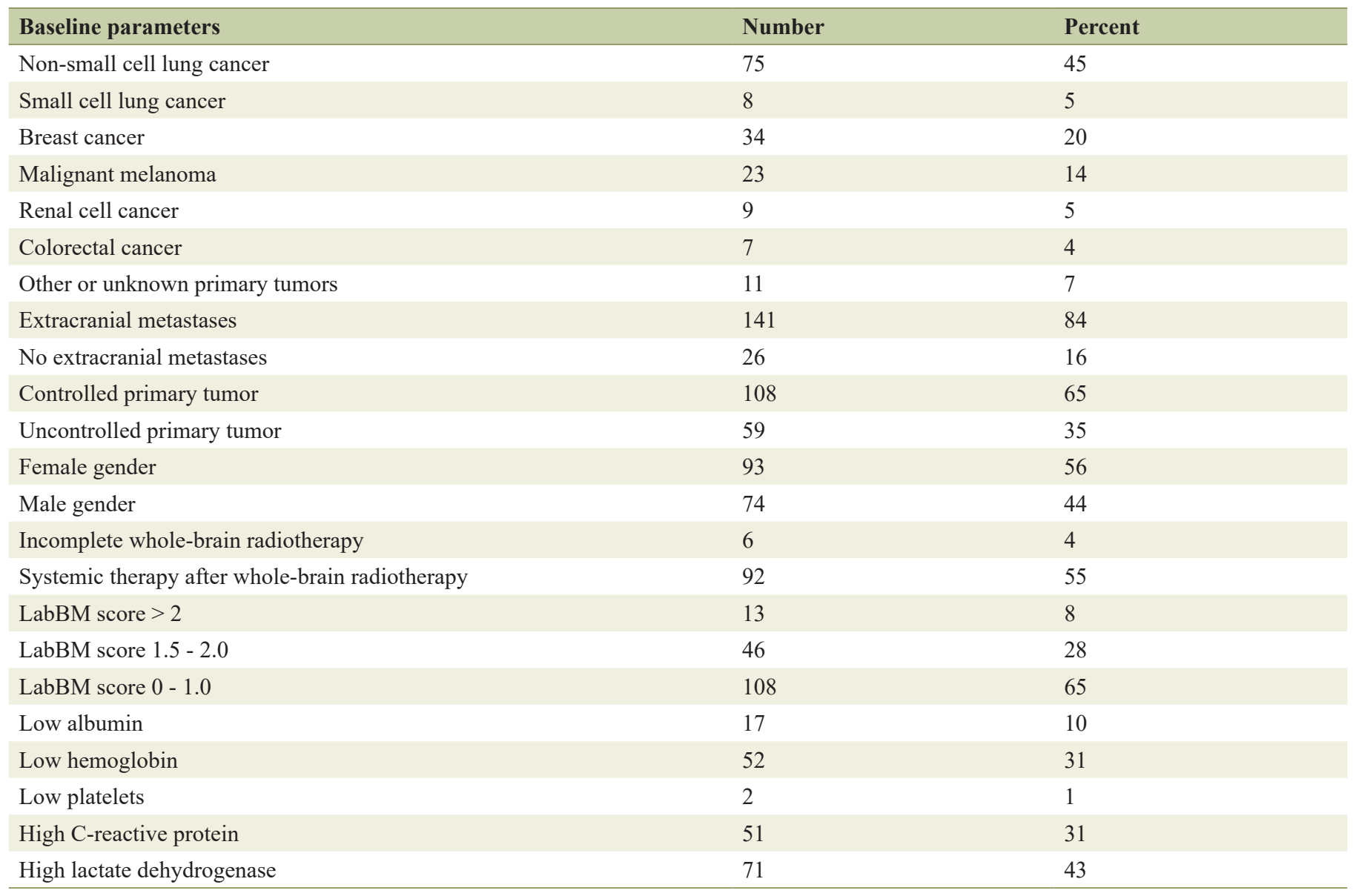

tween January 1, 2007 and December 31, 2017. They were discussed in a tumor board and found unsuitable for SRS or resection. Extracranial staging consisted of computed tomography $(\mathrm{CT})$. If clinically relevant, other staging examinations were added to clarify CT findings, for example, isotope bone scan, ultrasound, etc. All blood tests needed to calculate the LabBM score were routinely assessed at the start of WBRT or within the preceding week (normal values: hemoglobin 11.7 $15.3 \mathrm{~g} / \mathrm{dL}$ (females) and 13.4 - $17.0 \mathrm{~g} / \mathrm{dL}$ (males); platelets 130 - $400 \times 10^{9}$; albumin 34 - $45 \mathrm{~g} / \mathrm{L} ; \mathrm{LDH}<255 \mathrm{U} / \mathrm{L} ; \mathrm{CRP}<5$ $\mathrm{mg} / \mathrm{L})$. The score was calculated as described by Berghoff et al [9]. Briefly, 1 point was given for LDH and CRP measurement above the upper limit of normal and 0.5 points for hemoglobin, platelets and albumin below the lower limit of normal. A point sum of 0 indicates a favorable prognosis. The maximum point sum is 3.5 .

Overall survival (time to death) from the first day of radiotherapy was calculated employing the Kaplan-Meier method, and different groups were compared using the log-rank test (SPSS 25, IBM Corp., Armonk, NY, USA). Only five patients were censored after median 60 months of follow-up (minimum 2 months). Date of death was known in all other patients. In addition, the LabBM was evaluated by forward conditional Cox regression analysis (continuous variable, range $0-3.5$ points) [12]. Statistical significance was defined as $\mathrm{P}<0.05$ throughout this study.

This article does not contain any prospective studies with human participants or animals performed by any of the authors. The study was performed as a retrospective quality of care study and therefore no ethical committee, data inspectorate approval or informed consent was necessary, identical to our previous studies $[8,10,11]$. Consequently, no approval from the Regional Committees for Medical and Health Research Ethics (REK Nord) was necessary. Similarly, no approval from the Norwegian Social Science Data Services (NSD) was required.

\section{Results}

We identified and included 167 patients from the database. The largest group (45\%) had non-small cell lung cancer (NSCLC). The median age was 66 years (range: 35 - 88 years). The median Karnofsky performance status (KPS) was 70 (range: 30 - 100). According to the LabBM score, most patients $(65 \%)$ belonged to the group with favorable prognosis. Only $8 \%$ were assigned to the group with unfavorable prognosis. Further patient characteristics are shown in Table 1. 


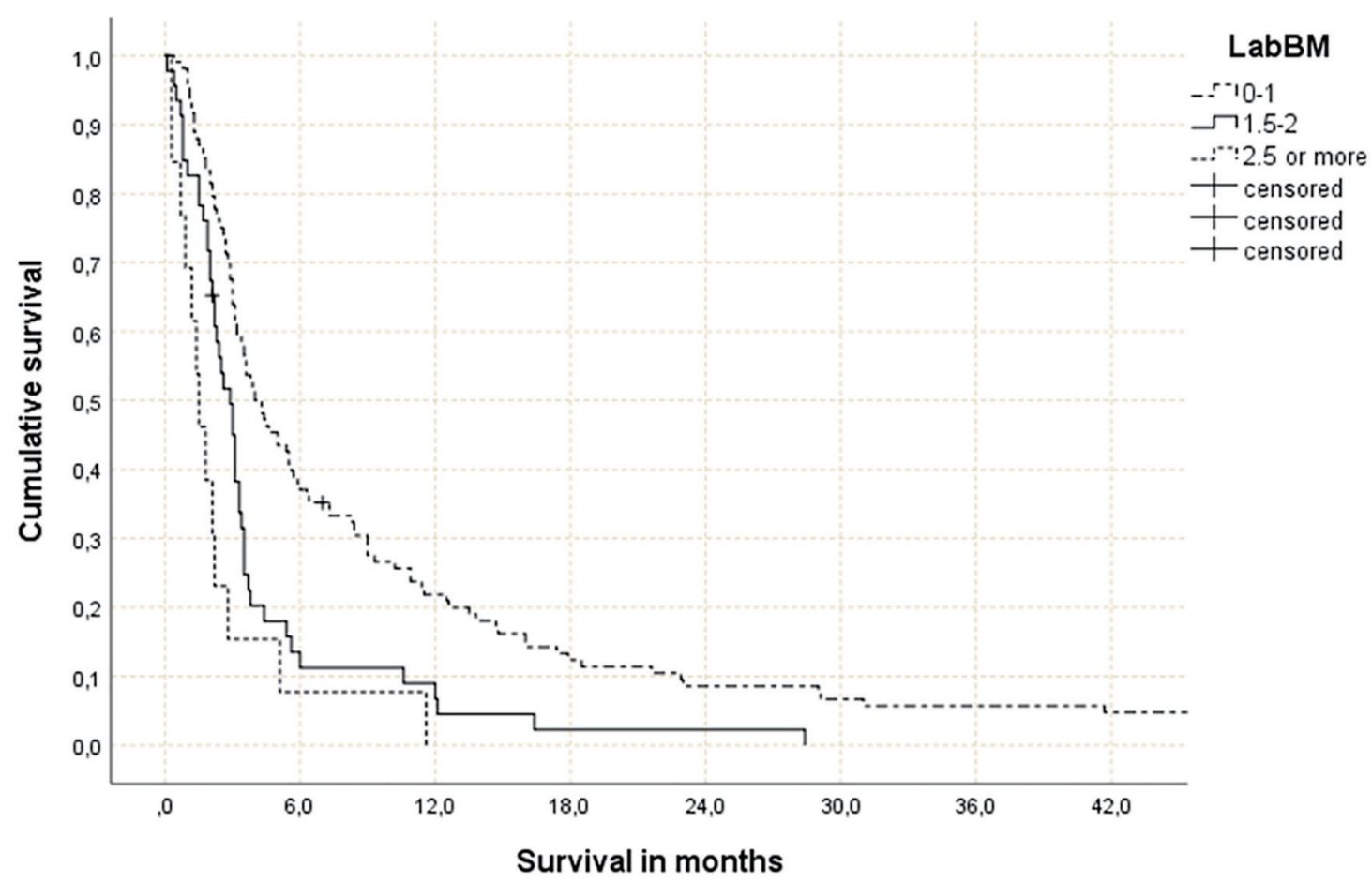

Figure 1. Actuarial overall survival stratified by LabBM score ( 0 - 1 point: median 4.0 months; 1.5 - 2 points: median 2.9 months; $>2$ points: median 1.5 months; $P=0.0001$ (best vs. intermediate and best vs. worst group), $P=0.046$ (intermediate vs. worst group)).

The median overall survival of all patients was 3.3 months ( $95 \%$ confidence interval (CI): 3.0 - 3.6 months). As shown in Figure 1, the LabBM score was significantly associated with survival. Median survival was 4.0 (CI: $2.9-5.1), 2.9$ (CI: 2.1 - 3.7) and 1.5 months (CI: $0.8-2.2$ ). All three statistical comparisons had $\mathrm{P}$ values $<0.05$ (0.046, 0.0001 and 0.0001 , respectively). In Cox regression analysis, the LabBM score was also significant (hazard ratio (HR): 1.87, CI: $1.46-2.40, \mathrm{P}=$ 0.0001 ). Among six patients who failed to complete WBRT, two each belonged to the three different LabBM classes. Table 2 shows an overview of the original and present LabBM results.

\section{Discussion}

We performed a retrospective study to test the validity of the LabBM score in patients treated with upfront WBRT rather than focal treatment such as SRS or surgical resection. Previously, many patients with more than 1 - 3 brain metastases received WBRT as their initial local treatment [13]. Based on the results of recent studies $[14,15]$, an increasing number

Table 2. Comparison Between the Three Datasets That Were Used to Develop and Validate the LabBM Score

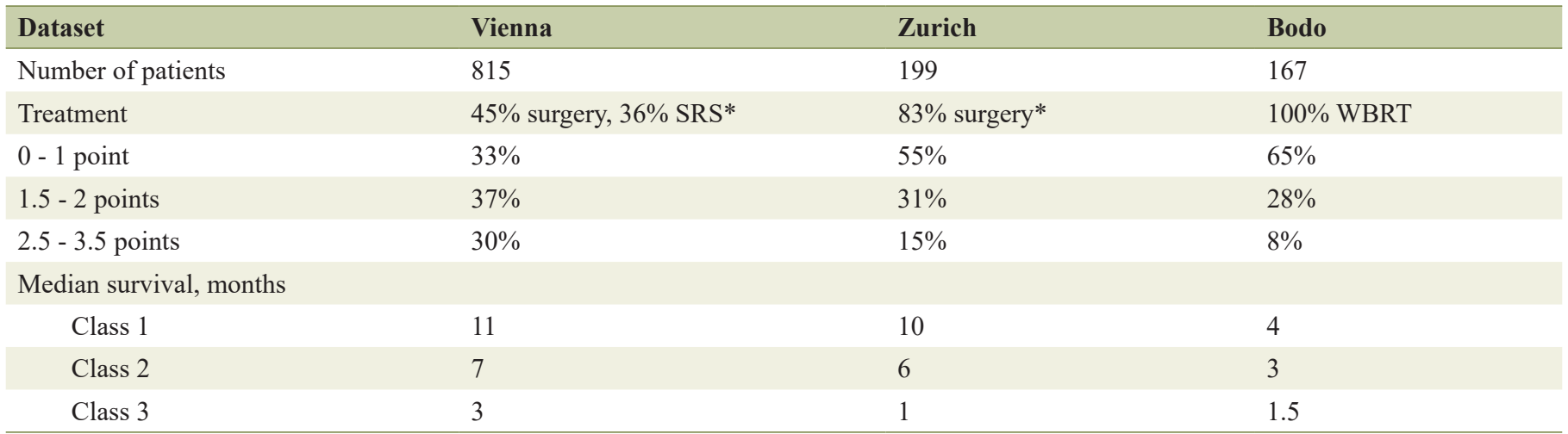

SRS: stereotactic radiosurgery; WBRT: whole-brain radiotherapy. *Percentage for 1,200 and 366 patients, respectively (not all had complete blood tests available). 
of patients are currently offered upfront SRS, and WBRT is often deferred. Nevertheless, WBRT continues to represent a management option for patients with a large number of brain metastases and/or location/size that render SRS or surgery difficult [16]. Furthermore, comorbidity, total tumor burden and compromised organ function might be arguments against focal treatment. An important aspect of decision-making is to avoid under-treatment in patients who require effective therapy to prolong their lives and to avoid overtreatment in patients with poor prognostic features. Survival prediction by use of nomograms and scores is therefore of high clinical relevance, although individual patients may live longer or shorter than predicted by the available models.

Our group has previously studied prognostic models that included tumor markers, albumin and $\operatorname{LDH}[8,11]$ and has suggested proceeding with additional research, because a combination of high LDH and low albumin often was associated with short survival. In a study limited to patients with renal cell cancer, Sperduto et al found that patients with low hemoglobin experienced shorter survival [17]. However, Berghoff et al expanded this strategy, which utilizes inexpensive standard test results, to include additional parameters (CRP and platelet count) and to validate the results in an independent external cohort [9]. Interestingly, their LabBM score is solely based on blood tests without including the traditional prognostic factors, for example, KPS or number of brain metastases. As discussed in their publication, the blood test results likely mirror and simplify a variety of other prognostic information, such as extracranial disease burden, total tumor burden, cachexia and systemic inflammatory processes. It appears nevertheless prudent to examine whether or not the LabBM score is a complete model where surrogate markers reflect the patients' performance status, etc., or if additional information may improve the score's ability to discriminate optimally between three different strata. The published survival curves for the unfavorable subset extended to beyond 12 months, despite short median survival ( 3 and 1 month in the development and validation cohort, respectively). Ideally, the unfavorable prognostic group would only contain patients with very short survival, because otherwise clinicians could be reluctant to withhold intense and/or costly brain-directed therapy (risk of overtreatment).

We also compared the original and present LabBM results, as displayed in Table 2. As expected from the differences in brain-directed treatment and patient selection, the original cohorts displayed longer survival, especially for patients with favorable and intermediate prognosis. There is striking disagreement in the proportion of patients with favorable and unfavorable prognosis (33-65\% and 8-30\%), which suggests a need to compare with additional studies. Nevertheless, the validity of the score could be confirmed. In our cohort, common abnormal blood tests included LDH, CRP and hemoglobin. Limitations of the present study include the limited number of patients, statistical power of subgroup analyses and retrospective design. Recently, separate prognostic scores have been advocated for patients with different primary tumors, for example, the lungmolGPA, melanoma-molGPA and renal GPA [7, 17, 18]. This diversification allows for inclusion of certain common molecular alterations, which alter systemic treatment paradigms.
It appears warranted to perform further optimization studies, either by adding the LabBM variables to those necessary to assign the diagnosis-specific prognostic assessment with the aim of developing LabGPA scores, or by creating new prognostic classes that integrate information from both scores (favorable LabBM and favorable lung-molGPA, unfavorable LabBM but intermediate lung-molGPA, etc.). The relatively large group of patients with lung cancer may be a suitable subset for a pilot study addressing these research avenues.

\section{Conclusion}

The LabBM score is also valid in a patient population that differs from the previously studied cohorts, that is, patients who were judged to be better candidates for WBRT than surgery or SRS. As these patients in general represent a less favorable subset, for example, regarding intracranial tumor volume, their median survival was shorter than reported in the development cohort (11, 7 and 3 months, respectively). Future studies should examine whether or not combinations of the LabBM and other scores, for example, lung-molGPA and melanomamolGPA, improve the clinical value of single scores.

\section{Acknowledgments}

None.

\section{Funding}

No funding was received for this study.

\section{Financial Disclosure}

None.

\section{Conflict of Interest}

The authors declare that they have no conflict of interest.

\section{Author Contributions}

$\mathrm{CN}$ analyzed and interpreted the patient data regarding the LabBM score. All authors contributed in writing the manuscript. All authors read and approved the final manuscript.

\section{References}

1. Franchino F, Ruda R, Soffietti R. Mechanisms and Therapy for Cancer Metastasis to the Brain. Front Oncol. 2018;8:161.

2. Nieder C, Mehta MP, Geinitz H, Grosu AL. Prognostic 
and predictive factors in patients with brain metastases from solid tumors: A review of published nomograms. Crit Rev Oncol Hematol. 2018;126:13-18.

3. Miyazawa K, Shikama N, Okazaki S, Koyama T, Takahashi T, Kato S. Predicting prognosis of short survival time after palliative whole-brain radiotherapy. J Radiat Res. 2018;59(1):43-49.

4. Rades D, Blanck O, Khoa MT, P VANT, Hung NQ, Dziggel L, Schild SE. Validation of a Survival Score for Patients Receiving Radiosurgery or Fractionated Stereotactic Radiotherapy for 1 to 3 Brain Metastases. In Vivo. 2018;32(2):381-384.

5. Rice SR, Bentzen SM, Hanna A, Choi E, Boggs DH, Kwok Y, Hyder J, et al. Prognostic models for patients with brain metastases after stereotactic radiosurgery with or without whole brain radiotherapy: a validation study. J Neurooncol. 2018;140(2):341-349.

6. Lagerwaard FJ, Levendag PC, Nowak PJ, Eijkenboom WM, Hanssens PE, Schmitz PI. Identification of prognostic factors in patients with brain metastases: a review of 1292 patients. Int J Radiat Oncol Biol Phys. 1999;43(4):795-803.

7. Sperduto PW, Yang TJ, Beal K, Pan H, Brown PD, Bangdiwala A, Shanley R, et al. Estimating Survival in Patients With Lung Cancer and Brain Metastases: An Update of the Graded Prognostic Assessment for Lung Cancer Using Molecular Markers (Lung-molGPA). JAMA Oncol. 2017;3(6):827-831.

8. Nieder C, Marienhagen K, Dalhaug A, Aandahl G, Haukland E, Pawinski A. Prognostic models predicting survival of patients with brain metastases: integration of lactate dehydrogenase, albumin and extracranial organ involvement. Clin Oncol (R Coll Radiol). 2014;26(8):447-452.

9. Berghoff AS, Wolpert F, Holland-Letz T, Koller R, Widhalm G, Gatterbauer B, Dieckmann K, et al. Combining standard clinical blood values for improving survival prediction in patients with newly diagnosed brain metastases-development and validation of the LabBM score. Neuro Oncol. 2017;19(9):1255-1262.

10. Nieder C, Hintz M, Oehlke O, Bilger A, Grosu AL. The TNM $8 \mathrm{M} 1 \mathrm{~b}$ and M1c classification for non-small cell lung cancer in a cohort of patients with brain metastases. Clin Transl Oncol. 2017;19(9):1141-1146.
11. Nieder C, Dalhaug A, Haukland E, Mannsaker B, Pawinski A. Tumor marker analyses in patients with brain metastases: patterns of practice and implications for survival prediction research. Tumour Biol. 2015;36(8):64716476.

12. Cox DR. Regression models and life tables. J Royal Stat Soc. 1972;34:187-220.

13. Tsao MN, Lloyd NS, Wong RK, Rakovitch E, Chow E, Laperriere N, the Supportive Care Guidelines Group of Cancer Care Ontario's Program in Evidence-based Care. Radiotherapeutic management of brain metastases: a systematic review and meta-analysis. Cancer Treat Rev. 2005;31(4):256-273.

14. Aoyama H, Tago M, Shirato H, for the Japanese Radiation Oncology Study Group 99-1 (JROSG 99-1) Investigators. Stereotactic radiosurgery with or without whole-brain radiotherapy for brain metastases: secondary analysis of the JROSG 99-1 randomized clinical trial. JAMA Oncol. 2015;1(4):457-464.

15. Soffietti R, Kocher M, Abacioglu UM, Villa S, Fauchon F, Baumert BG, Fariselli L, et al. A European Organisation for Research and Treatment of Cancer phase III trial of adjuvant whole-brain radiotherapy versus observation in patients with one to three brain metastases from solid tumors after surgical resection or radiosurgery: qualityof-life results. J Clin Oncol. 2013;31(1):65-72.

16. Nieder C, Guckenberger M, Gaspar LE, Rusthoven CG, De Ruysscher D, Sahgal A, Nguyen T, et al. Management of patients with brain metastases from non-small cell lung cancer and adverse prognostic features: multi-national radiation treatment recommendations are heterogeneous. Radiat Oncol. 2019;14(1):33.

17. Sperduto PW, Deegan BJ, Li J, Jethwa KR, Brown PD, Lockney N, Beal K, et al. Estimating survival for renal cell carcinoma patients with brain metastases: an update of the Renal Graded Prognostic Assessment tool. Neuro Oncol. 2018;20(12):1652-1660.

18. Sperduto PW, Jiang W, Brown PD, Braunstein S, Sneed P, Wattson DA, Shih HA, et al. Estimating survival in melanoma patients with brain metastases: an update of the graded prognostic assessment for melanoma using molecular markers (Melanoma-molGPA). Int J Radiat Oncol Biol Phys. 2017;99(4):812-816. 